\title{
Development of anisotropy in ultra-high molecular weight polyethylene
}

\author{
P. Gao, M. R. Mackley* and T. M. Nicholson \\ Department of Chemical Engineering, University of Cambridge, Pembroke Street, \\ Cambridge CB2 3RA, UK
}

(Received 1 February 1989; revised 26 April 1989; accepted 5 June 1989)

\begin{abstract}
We report experimental measurements on the drawing behaviour of a pre-swollen drawn sample of ultra-high molecular weight polyethylene (UHMWPE). X-ray information is expressed in terms of an orientation function $P_{2}(\theta)$, and the increase in both $P_{2}$ and the tensile modulus are determined as a function of draw ratio. Scanning electron microscopy is used to follow the structural changes in the material. The experimental results are compared with a numerical simulation of the deformation and from these we suggest that early stages of deformation involve crystal orientation followed by subsequent chain extension at higher draw ratios.
\end{abstract}

(Keywords: swelling; ultrahigh molecular weight polyethylene; $\mathrm{X}$-ray analysis high modulus; mechanical properties; aggregate model)

\section{INTRODUCTION}

Within the last ten years a number of related processing routes have been discovered for manufacturing high strength and stiffness polyethylene structures using ultra-high molecular weight polyethylene (UHMWPE) precursor material. Zwijnenburg and Pennings ${ }^{1}$ developed a surface growth process where the fibres were crystallized and drawn directly from a polyethylene solution. Smith and Lemstra ${ }^{2}$ pioneered the gel spinning technology where a 'low entanglement' gel fibre is spun and then crystallized; the solvent is removed and anisotropy generated in the fibre by means of hot drawing. Other process routes have been reported which include variants of gel spinning ${ }^{3,4}$, ram extrusion of 'low entanglement' UHMWPE ${ }^{5-7}$, drawing of reactor UHMWPE material ${ }^{8,9}$, die free spinning ${ }^{10}$ and the swell drawing of UHMWPE ${ }^{11}$. The swell draw process involves starting with an isotropic solid piece of material. The polymer is then swollen using an appropriate solvent, temperature and time such that high swelling ratios are achieved without the material losing its mechanical integrity. In the swollen state it is assumed that the level of entanglement within the material is reduced. The low entanglement concentration is further assumed to be trapped within the material when the swollen material is quenched and crystallization occurs. After solvent removal, anisotropy is developed by hot drawing the material. It has been found that the pre-swelling of the polymer greatly enhances the material's ability to be drawn above its natural draw ratio ${ }^{11}$ and correspondingly the tensile properties are also enhanced ${ }^{7}$.

The primary purpose of this paper is to describe experimental observations we have made on a swell drawn UHMWPE sample and discuss these results in terms of the possible deformation mechanisms occurring within the material at different stages in the drawing process. A number of authors have attempted to describe the deformation behaviour of polymers ${ }^{12,13}$ and recently Termonia and Smith ${ }^{14}$ have developed a numerical simulation of drawing behaviour which covers drawing

\footnotetext{
* To whom correspondence should be addressed
}

both for normally 'entangled' and 'low entanglement' materials.

\section{EXPERIMENTAL}

Swelling, quenching, crystallization and solvent removal

The polymer used in this study was UHMWPE HG415 manufactured by Hoechst. Tapes of $0.5 \mathrm{~mm}$ thickness were used and they were supplied by Performance Plastic Ltd (Bacup, UK) from slabs that had been prepared by ram extrusion. Specimens were annealed for $25 \mathrm{~min}$ at $150^{\circ} \mathrm{C}$ and oven cooled to relieve any residual thermal and mechanical history. Swelling was done by immersing preweighed specimens in a solvent bath of decalin which was maintained at the appropriate temperature by a silicon oil bath. After chosen times, specimens were removed from the bath and weighed with a microbalance which was connected to a chart recorder. The weight was measured as a function of time and the total swollen weight obtained by extrapolating the curve to zero time (solvent loss between immersion and weighing was $<1 \%$ ). Figure 1 shows the observed weight uptake for decalin solvent as a function of time; the experiments were conducted at $125^{\circ} \mathrm{C}$. The experimental data is plotted in terms of $W_{\mathrm{s}} / W_{\mathrm{p}}$ as a function of time, where $W_{\mathrm{s}}$ is the solvent weight within the polymer at time $t$ and $W_{\mathrm{p}}$ the initial weight of the polymer sample. The plot shows an essentially linear weight uptake as a function of time and the ability to achieve high solvent loadings is apparent.

Quenching of the swollen samples was achieved by removing the sample from the bath and cooling in the ambient conditions. We estimate that the material would have cooled from the bath temperature of $125^{\circ} \mathrm{C}$ to an appropriate crystallization temperature of say $80^{\circ} \mathrm{C}$ within $5 \mathrm{~s}$. The crystallized gel samples were left to dry at ambient conditions for $\approx 12 \mathrm{~h}$, and then they were transferred to a $\mathrm{JJ}$ environmental cabinet and held at $80^{\circ} \mathrm{C}$ for $\approx 20 \mathrm{~min}$ to enhance solvent evaporation. The nearly dried gel films were finally immersed in an excess of acetone and subsequently air dried to remove residual 


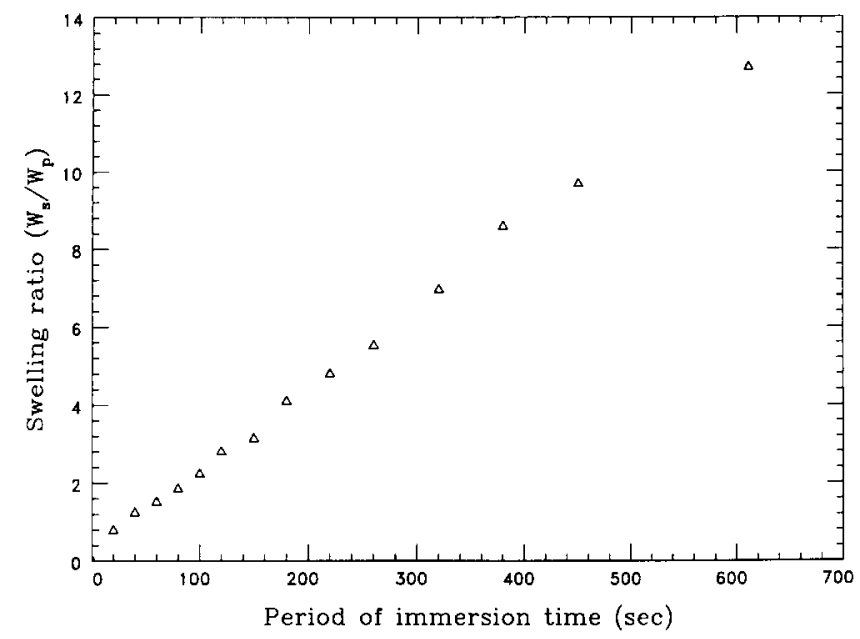

Figure 1 Solvent weight uptake versus immersion time for $0.5 \mathrm{~mm}$ thick HG415 tapes in decalin; swelling temperature $125^{\circ} \mathrm{C}$

traces of decalin-acetone mixture. We estimate that before drawing the residual solvent content was $<2 \%$. The dried gel films were cut into dumb-bell shaped specimens with a gauge length of $25 \mathrm{~mm}$ and a width of $4 \mathrm{~mm}$ for the drawing experiment.

\section{Drawing}

The hot drawing behaviour of the samples was studied using a JJ tensile drawing testing machine equipped with an environmental cabinet. Drawing was performed under isothermal conditions at a constant crosshead speed of $100 \mathrm{~mm} \mathrm{~min}^{-1}$. Draw ratios $\lambda$ were determined in the usual way by measuring the displacement of ink marks placed $20 \mathrm{~mm}$ apart on the specimen before drawing. Elongation of $\lambda>10$ was carried out as a multi-stage process, because of a length constraint of the environmental cabinet. The specimen was drawn to $\lambda=10$ in the first stage and the drawn film was then cut into strips of length of $80-90 \mathrm{~mm}$. These specimens were subsequently clamped over a length of $50 \mathrm{~mm}$ and further drawn to about $200 \mathrm{~mm}$ in a second stage. In this way draw ratios between $\lambda=10$ and $\lambda=40$ could be explored. Results reported in this paper relate to a single initial swelling condition of decaline immersion for $440 \mathrm{~s}$ at $125^{\circ} \mathrm{C}$. These samples were then drawn to different draw ratio at $120^{\circ} \mathrm{C}$. Under these conditions it was found that the maximum draw ratio that could be achieved was 40 .

\section{Scanning electron microscopy (SEM) observation}

SEM photographs of the free surface of the swell drawn material are shown in Figure 2. Figure $2 a$ shows the undrawn pre-swollen starting material. The material shows evidence of porosity and unoriented lamellar crystals appear to be present. We saw no direct evidence to suggest that spherulitic crystallization had occurred. At a modest draw ratio of 5 , as shown in Figure $2 b$, a transition between a lamellar and a fibrillar texture appeared to be occurring. At high draw ratios, as shown in Figures $2 c$ and $d$, the fibrillar texture dominated, with the orientation and clarity of the fibrous entities increasing with increasing draw ratio.

\section{$X$-ray measurements}

Wide angle $\mathrm{X}$-ray diffraction (WAXD) patterns were obtained using a flat film camera and $\mathrm{CuK}_{\alpha}$ radiation at $30 \mathrm{~mA}$ and $40 \mathrm{kV}$. The $\mathrm{X}$-ray beam was made monochromatic by using a nickel filter to give a wavelength of $0.15417 \mathrm{~nm}$. The exposure time for all the specimens was $25 \mathrm{~min}$, and the working distance between sample and film was $30 \mathrm{~mm}$. Figure 3 shows the evolution of the $\mathrm{X}$-ray diffraction pattern as a function of draw ratio. Figure $3 a$ shows the typical polyethylene diffraction rings for unoriented material. Drawing induces molecular orientation, which leads to the equatorial concentration of scattering intensity for the 200,020 and 110 diffraction peaks, as shown in Figure $3 c$ and $d$.

The variation of intensity around the appropriate arc of the diffraction pattern was determined by using an image analyser. The relative intensity around arcs (200) and $(020)$ (i.e. $I(\phi)_{200}$ and $\left.I(\phi)_{020}\right)$ was obtained by a scan determining the scattering intensity $I(\phi)$ at a position $(x, y)$ on the photograph negative, where $x^{2}+y^{2}=R^{2}$ and $R$ is the radius of the diffraction arc. The intensity was measured at $1^{\circ}$ increments around the arcs and the value of the orientation function $P_{2}(\theta)$ was computed from the intensity measurements using the method described by Stein ${ }^{15}$, which is summarized in the Appendix to this paper.

\section{Mechanical properties measurements}

The Young's modulus and strength of the drawn tapes were measured at room temperature $\left(\approx 20^{\circ} \mathrm{C}\right)$. Samples of initial length $150-170 \mathrm{~mm}$ and a crosshead speed of $100 \mathrm{~mm} \mathrm{~min}^{-1}$ were used, and measurements were taken at the point of the maximum slope on the load-deflection graph. Errors in each measurement are estimated to be of the order of $\pm 10 \%$.

\section{MODELLING DRAW BEHAVIOUR}

We have chosen to simulate the drawing behaviour of the pre-swollen polymer by using the aggregate model and pseudo-affine deformation, which has been successfully used by other workers to describe the deformation of melt crystallized semicrystalline polymers ${ }^{12,16,17}$. In the aggregate model the material is assumed to be composed of an aggregate of elastically anisotropic units. Each of these units is assigned elastic properties which are assumed to be independent of deformation. For any given distribution of orientations of these units the orientation function and the tensile modulus may be calculated.

Initially a random distribution of orientations is generated, corresponding to the undrawn polymer. The draw process is then modelled by applying a pseudoaffine deformation, the orientation in each unit being altered as if the vectors describing these orientations were embedded in a substrate that is uniformly drawn without change of volume (Figure 4). Changes in the length of the vectors are ignored; only the resulting directions are considered. At each draw ratio, the orientation function and tensile modulus were then calculated from the distribution of unit orientations. The same results were obtained regardless of whether the orientation distribution was generated by applying a single step deformation, or as the product of several smaller draw deformations. An array of 2000 units was used in the simulations described here.

The orientation function corresponding to a given distribution can be calculated simply by computing the 

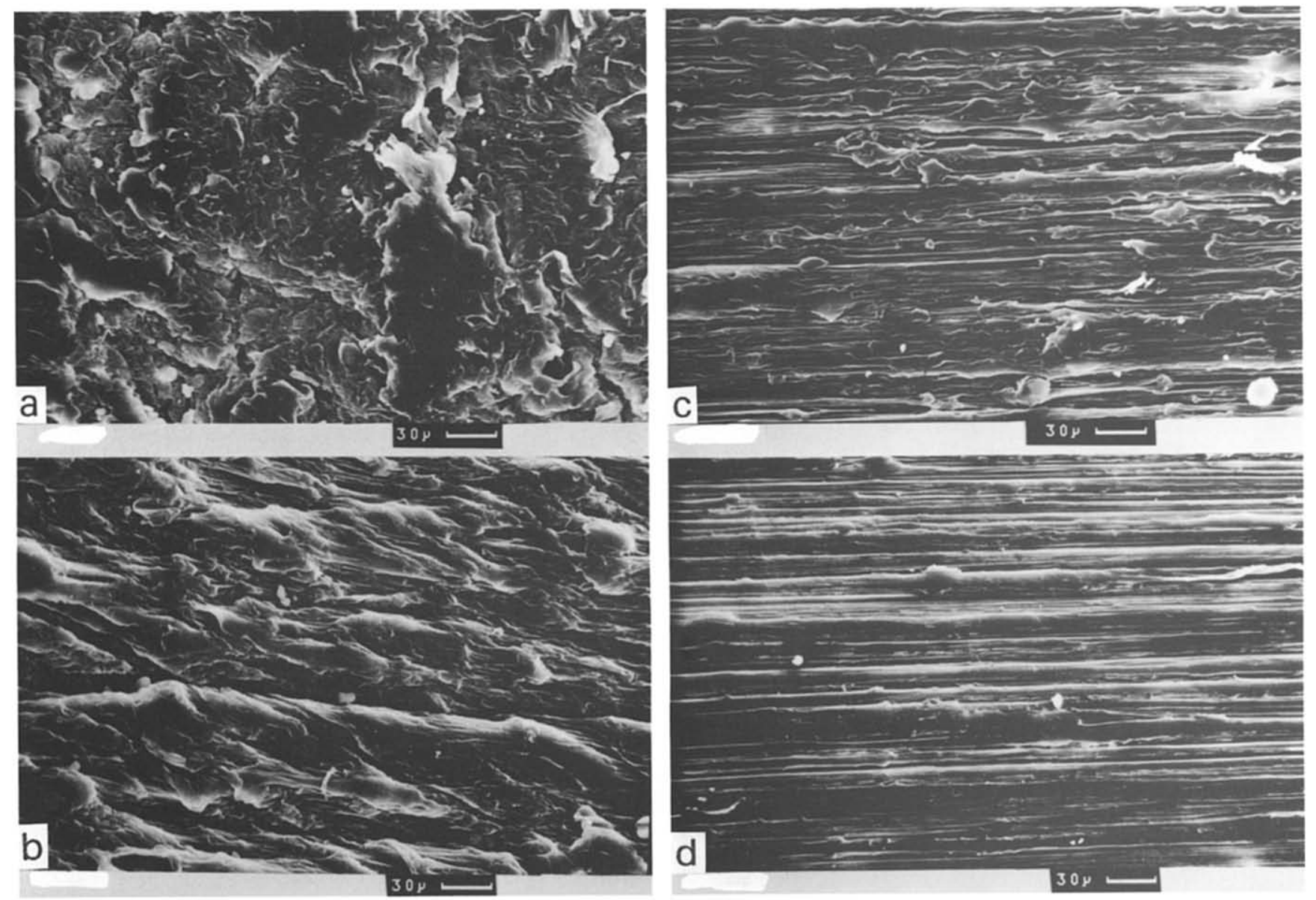

Figure 2 Scanning electron micrographs of drawn UHMWPE films with draw ratios $\lambda$ of (a) 1.0 , (b) 5.0 , (c) 15.0 and (d) 40.0. The stretching direction is horizontal

average

$$
P_{2}=\frac{1}{2}\left\langle 3 \cos ^{2} \alpha-1\right\rangle
$$

where $\alpha$ is the angle between the orientation of a particular cell and the draw direction and the angle brackets indicate averaged quantities. Results from such calculations are shown in Figure 5, superimposed on the experimental data obtained by analysis of the X-ray results. A striking agreement is obtained between the experimental data and the numerical prediction.

The tensile modulus is calculated by averaging elastic constants throughout the aggregate. This may be done in two limiting ways ${ }^{16}$, either by assuming a uniform stress throughout the material (effectively placing the units in series) or by assuming a uniform strain (corresponding to a parallel coupling of the units).

The first method involves an averaging of compliance coefficients. Using tensor transformations, the extensional compliance along the draw direction in each unit, $s_{33}^{\prime}$, may be calculated in terms of the compliance coefficients along the major axes of a unit cell, which in turn are related to physical properties of the highly oriented polymer (Table 1). The extensional strain in this unit $e$ is related to the stress $\sigma$ by the expression $e=s_{33}^{\prime} \sigma$. Averaging over all the units gives $\langle e\rangle=\left\langle s_{33}^{\prime}\right\rangle \sigma$ and hence the tensile modulus $E$ is given by

$$
E=\frac{\sigma}{\langle e\rangle}=\frac{1}{\left\langle s_{33}^{\prime}\right\rangle}
$$

The alternative method of calculating the tensile modulus for a given distribution of unit orientations involves an averaging of stiffness coefficients and leads to a second expression for the tensile modulus:

$$
E=\left\langle c_{33}^{\prime}\right\rangle
$$

where $c_{33}^{\prime}$ is the extensional stiffness along the draw direction of a particular unit. This can be calculated again by tensor methods from the principle stiffness coefficients for a unit cell. These stiffness coefficients are related numerically to the compliance coefficients.

These two methods yield an upper and lower bound ${ }^{18}$ for the mechanical response. The behaviour of a real material would be expected to fall between these bounds

A set of values of the physical properties for the highly oriented polymer is required to calculate the compliance coefficients for a unit cell. Since we have not performed definitive experiments ourselves to obtain the full set of properties, we used the published values ${ }^{12}$ for ultra-high modulus PE which are listed in Table 1 (column A).

The modulus predictions from data $A$ are shown in Figure 6, where both the compliance and the stiffness calculations are superimposed on the experimental tensile modulus data. In this case much of the experimental data falls outside the bounds imposed by the theoretical curves, and the predicted value for the isotropic polymer $(\lambda=1)$ is between 3 and $20 \mathrm{GPa}$, compared to an experimental observed value of $1.2 \mathrm{GPa}$.

A modified set of physical parameters was also used 

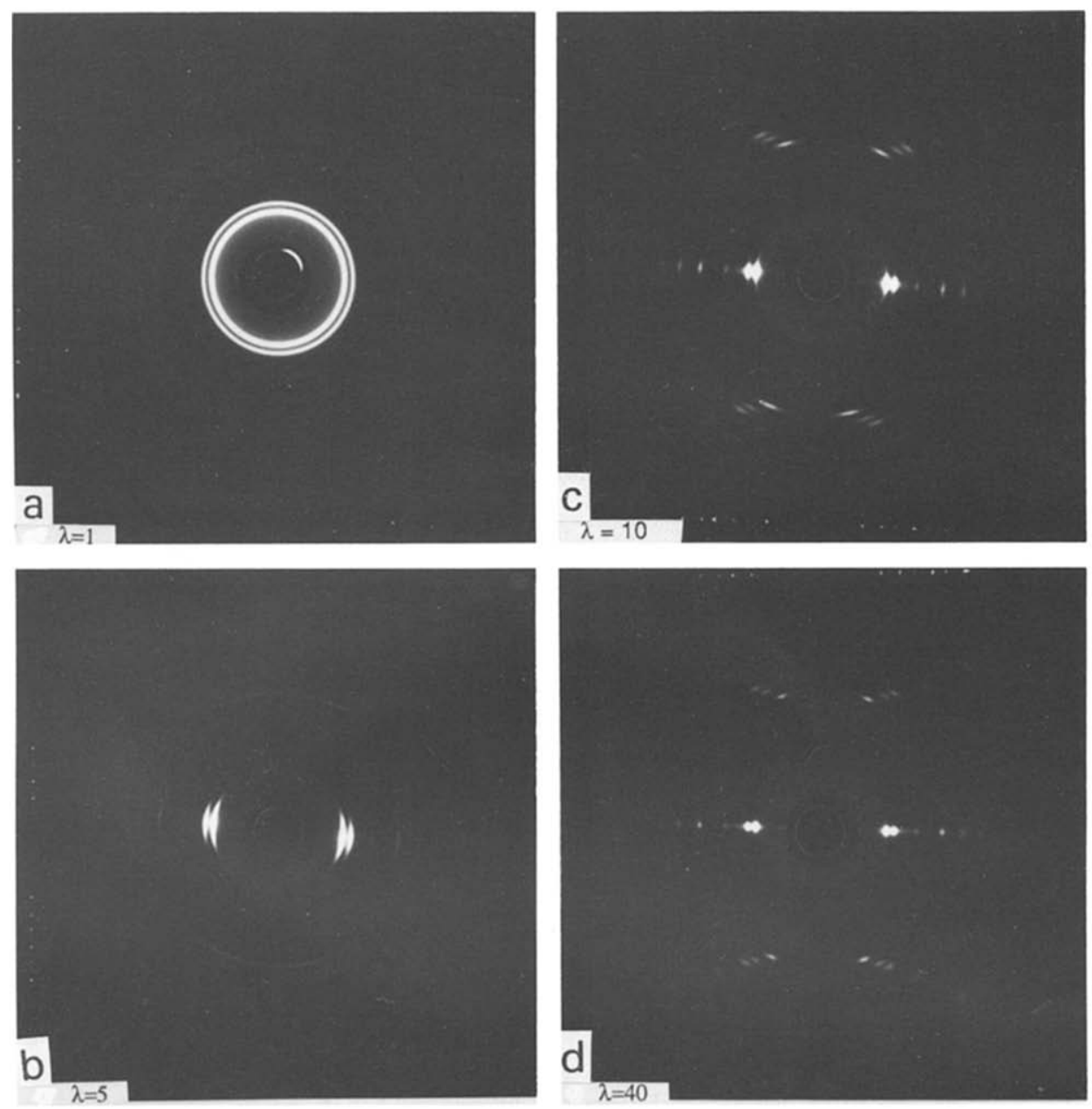

Figure 3 Wide angle X-ray photographs of pre-swollen drawn UHMWPE with draw ratios $\lambda$ of (a) 1.0 , (b) 5.0, (c) 10.0 and (d) 40.0. The drawing direction is vertical
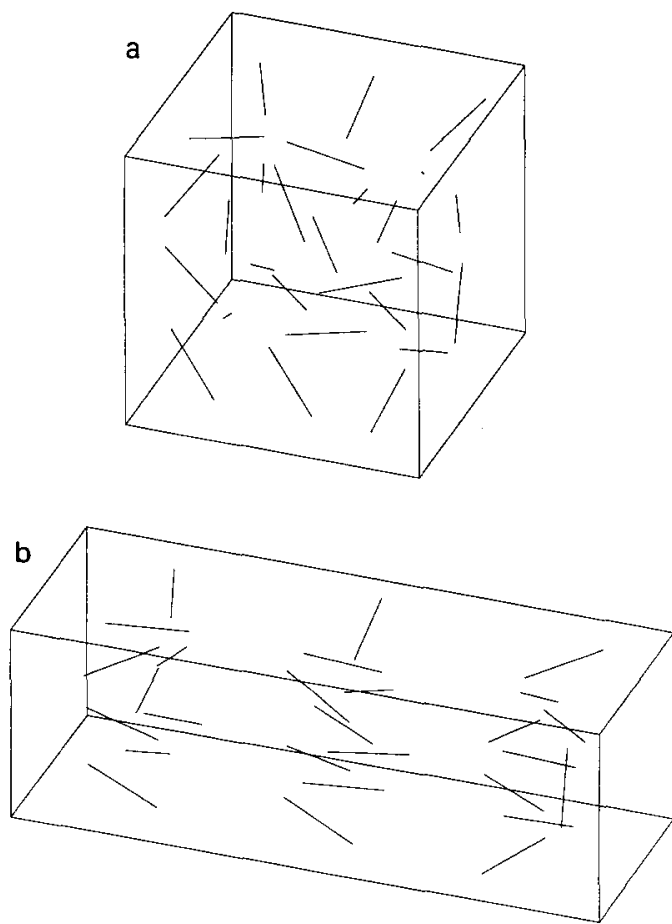

Figure 4 Schematic diagram showing the pseudo-affine deformation applied to the orientations of units in the aggregate: (a) initial stage, with random orientations; (b) after a draw ratio of 2 has been applied

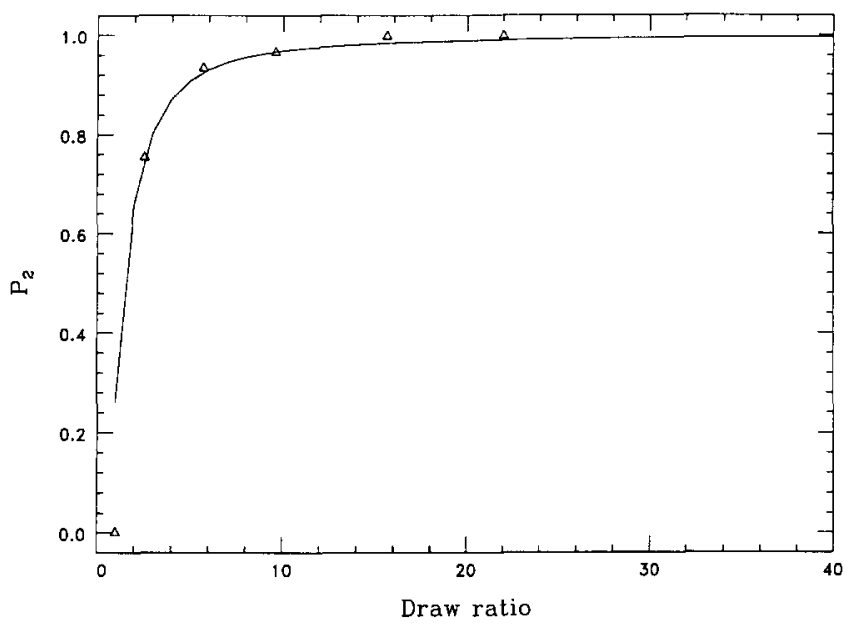

Figure 5 Orientation function as calculated from $X$-ray diffraction data and calculated using an aggregate model. $\triangle$, Experimental data; - calculated data

(Table 1, column B). $E_{\text {long }}$ was chosen to correspond to the tensile modulus of the highest drawn sample that we generated $(\lambda=42) . E_{\text {tran }}$ and $G$ were then calculated so that the predicted values of the isotropic moduli, $E_{\text {iso }}$ and $G_{\text {iso }}$, obtained using averaging of compliance coefficients were equal to the experimental values measured from undrawn samples $\left(E_{\text {iso }}=1.2 \mathrm{GPa}, G_{\text {iso }}=0.38 \mathrm{GPa}\right)$. It was not possible to obtain realistic values of $E_{\text {tran }}$ and $G$ by averaging stiffness coefficients. 
Table 1 Values of physical constants used in the simulations shown in Figures 6 and $7^{a}$

\begin{tabular}{lllcc}
\hline & & & $A^{b}$ & $B^{c}$ \\
\hline $\begin{array}{c}\text { Tensile modulus perpendicular } \\
\text { to symmetry axis (GPa) }\end{array}$ & $E_{\text {tran }}$ & $1 / s_{11}$ & 1.35 & 2.5 \\
$\begin{array}{c}\text { Tensile modulus parallel to } \\
\text { symmetry axis }(\mathrm{GPa})\end{array}$ & $E_{\text {long }}$ & $1 / s_{33}$ & 100 & 105 \\
Shear modulus $(\mathrm{GPa})$ & $G$ & $1 / s_{44}$ & 1.3 & 0.2 \\
Poisson's ratio & $v_{13}$ & $-s_{13} / s_{33}$ & 0.5 & 0.5 \\
Poisson's ratio & $v_{12}$ & $-s_{12} / s_{11}$ & 0.5 & 0.5 \\
& & & &
\end{tabular}

${ }^{a}$ Room temperature values quoted, $T=20^{\circ} \mathrm{C}$

${ }^{b}$ Values used for Figure 6 obtained from Reference 12

'Values used for Figure 7, adjusted as described in the text

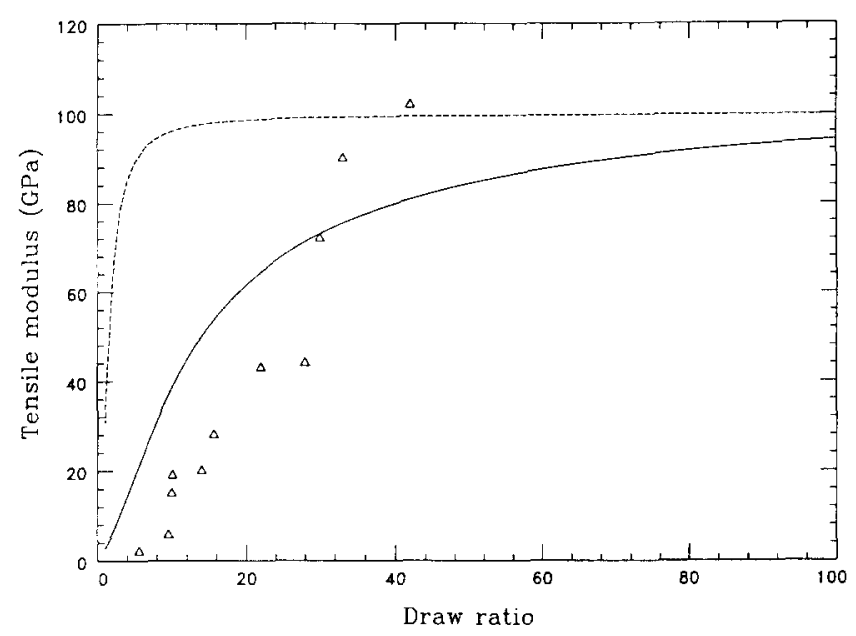

Figure 6 Tensile modulus as experimentally measured $(\triangle)$ and as calculated by an aggregate model, using compliance data derived from physical constants given in Reference 12 (Table 1, column A). The two theoretical curves correspond to the averaging of either compliance $(-)$ or stiffness coefficients $(--)$

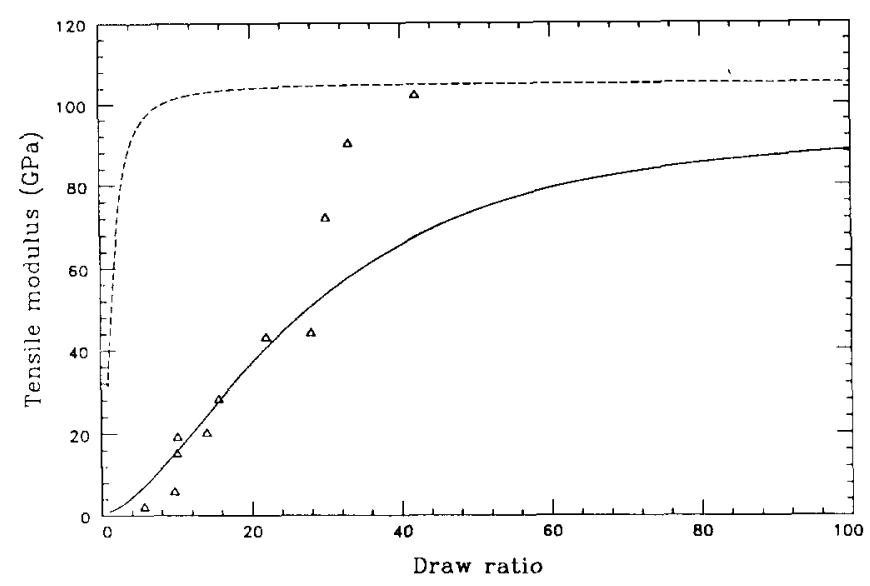

Figure 7 Tensile modulus as experimentally measured $(\Delta)$ and as calculated by an aggregate model, using compliance data derived from modified physical constants as described in the text (Table 1, column B). The two theoretical curves correspond to the averaging of either compliance ( …) or stiffness coefficients $(--)$
Theoretical curves obtained from these modified data are shown in Figure 7, superimposed on the experimental data. It can be seen that the observed mechanical properties follow the lower (compliance average) curve at low draw ratios and then move towards the upper (stiffness average) curve at higher draw ratios. The actual form of the theoretical curves was found to be sensitive to the chosen values of $E_{\text {tran }}$ and $G$ but essentially insensitive to varying Poisson ratios.

\section{DISCUSSION}

The experimental findings reported in this paper support the results presented in the initial publication on the swelling drawing process ${ }^{11}$ and show some similarities to results published on gel drawn UHMWPE fibres ${ }^{12}$. Molecular orientation measured from X-ray data increases with increasing draw ratio and appears to follow closely that expected from the pseudo-affine deformation of an aggregate of crystals. Most of the orientation is achieved in the early stages of deformation, say up to a draw ratio of order 3-4. Beyond this point $P_{2}(\theta)$ changes very little. The fact that the simulated value of $P_{2}(\theta)$ fits the experimental data so well is at first sight a surprising result because it is clear, for example from the SEM observations, that major structural changes are occurring during the deformation process.

The mechanical property data, when compared with simulated values in Figures 6 and 7, suggest that the material properties change from the lower bound behaviour to upper bound behaviour with the imposition of mechanical drawing. The initial lower bound behaviour suggests that the anisotropic units are coupled in a series sense with stress continuity at low deformations and are coupled in a parallel sense with strain continuity at high deformation levels.

The combined orientation and mechanical results suggest to us that at low deformations the dominant effect is crystal orientation, while at high deformations the changing mechanical properties suggest that chain extension rather than orientation is occurring. A schematic interpretation of events that could occur on drawing is shown in Figure 8. Clearly the detailed mechanisms associated with the drawing process are more complicated than drawn in the diagram and the additional aspect that the material becomes highly fibrillar also needs to be taken into account. However, the results do suggest that the enhanced drawability associated with preswollen UHMWPE is mainly connected with a chain extension process rather than chain orientation at the later stages of drawing.

The pseudo-affine deformation model appears to be surprisingly successful in describing the drawing behaviour of pre-swollen UHMWPE samples. The fitting

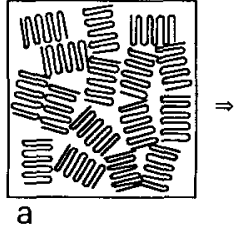

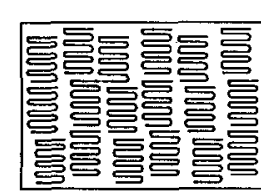

b

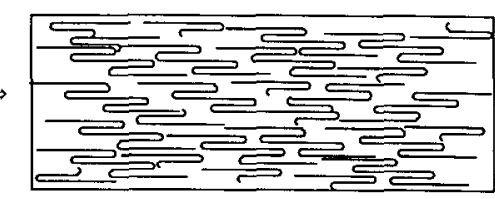

c

Figure 8 Schematic diagram showing suggested behaviour of swell-drawn polyethylene (a) Initially the polymer consists of a random arrangement of crystallites. (b) At low draw ratios these crystallites align along the draw direction, leading to an improved orientation function. (c) At higher draw ratios the polymer chains extend along the draw direction, increasing the tensile modulus, but not affecting the orientation function. 
of the aggregate model is sensitive to the values chosen for the elastic constants of the anisotropic units and at present there is some uncertainty on the exact values that should be chosen. When this issue has been resolved a more detailed analysis may be possible. Our scanning electron microscopy results show clearly that a major change in morphology occurs during deformation and the development of fibrillar structure in the draw direction becomes dominant at high draw ratios. This, together with an anticipated increase in crystallinity within the material, can be expected to make accurate molecular modelling of the mechanical drawing process complicated and difficult. It could in principle be possible to introduce some of these factors into the aggregate modelling but at this stage the introduction of further adjustable parameters seems unjustified.

\section{REFERENCES}

1 Zwijnenburg, A. and Pennings, A. J. Colloid Polym. Sci. 1976, 254, 868

2 Smith, P. and Lemstra, P. J. J. Mater. Sci. 1980, 19, 505

3 Lemstra, P. J., Van Aerle, N. A. J. M. and Bastiansen, C. W. M. Polym. J. 1987, 19, 85

4 Kirschbaum, R., Van Dingenrn, J. L. J. 'Integration of Polymer Sci. and Technology', Proceedings of Rolduc Conference, Elsevier Applied Sciences, London, 1988

5 Anton, C., Mackley, M. R. and Solbai, S. B. Polym. Bull. 1987, 17,175

6 Kanamoto, T., Tsurata, A., Tanaka, K., Takeda, M. and Porter, R. Macromolecules 1988, 21, 470

7 Kanamoto, T., Ohama, T., Tanaka, K., Takeda, M. and Porter, R. Polymer 1987, 28, 1517

8 Ohama, T., Kanamoto, T., Takeda, M. and Porter, R. Rep. Polym. Phys. Jpn 1987, 325-328

9 Pawlikowski, G. T., Mitchell, D. J. and Porter, R. S. J. Polym. Sci. Phys. 1988, 26, 1865

Mackley, M. R. and Solbai, S. B. Polymer 1987, 28, 1111

Mackley, M. R. and Solbai, S. B. Polymer 1987, 28, 1115

Ward, I. M. Adv. Polym. Sci. 1985, 70, 12

Peterlin, A. Colloid Polym. Sci. 1987, 265, 357

Termonia, Y. and Smith, P. Macromolecules 1988, 21, 2184

Stein, R. S. J. Polym. Sci. 1958, 21, 327

Ward, I. M. 'Mechanical Properties of Solid Polymers', Wiley, 1971, Ch. 10

17 Raumann, G. and Saunders, D. W. Proc. Phys. Soc. 1961, 77, 1028

18 Bishop, J. and Hill, R. Phil. Mag. 1951, 42, 414, 1248

\section{APPENDIX}

The crystal orientation functions are defined as:

$$
\begin{aligned}
& f_{\alpha}=\frac{1}{2}\left\langle 3 \cos ^{2} \alpha-1\right\rangle \\
& f_{\beta}=\frac{1}{2}\left\langle 3 \cos ^{2} \beta-1\right\rangle \\
& f_{\varepsilon}=\frac{1}{2}\left\langle 3 \cos ^{2} \varepsilon-1\right\rangle
\end{aligned}
$$

where $\alpha, \beta$, and $\varepsilon$ are the angles measured between the $z$ axis (the stretching direction) and the $a, b$ and $c$ crystallographic axes, respectively. $f_{\varepsilon}$ is normally used for describing crystal orientation function, i.e. $P_{2}(\theta)$.

To determine the $f$ s from the X-ray pattern, it is most convenient to work with the 200 and 020 reflections. The orientations of the crystals giving rise to the diffraction at these points are given by equations

$$
\cos \alpha=\cos \theta_{200} \sin \phi_{200}
$$

and

$$
\cos \beta=\cos \theta_{020} \sin \phi_{020}
$$

where $\theta_{200}$ and $\theta_{020}$ are the Bragg angles for the 200 and 020 reflections. The angles $\phi_{200}$ and $\phi_{020}$ are measured from the equator to a point on the two arcs.

Substituting equations (A4) and (A5) into equations (A1) and (A2), one obtains:

$$
\begin{aligned}
& f_{\alpha}=\frac{1}{2}\left(3 \cos ^{2} \theta_{200} \overline{\sin ^{2} \phi_{200}}-1\right) \\
& f_{\beta}=\frac{1}{2}\left(3 \cos ^{2} \theta_{020} \overline{\sin ^{2} \phi_{020}}-1\right)
\end{aligned}
$$

The values of $\overline{\sin ^{2} \phi}$ may be computed from the following equation:

$\overline{\sin ^{2} \phi_{200}}=\frac{\int_{0}^{\pi / 2} I\left(\phi_{200}\right) \sin ^{2} \phi_{200} \cos \phi_{200} \mathrm{~d} \phi_{200}}{\int_{0}^{\pi / 2} I\left(\phi_{200}\right) \cos \phi_{200} \mathrm{~d} \phi_{200}}$

where $I\left(\phi_{200}\right)$ is the relative intensity around arc (200) and was experimentally determined as described in this paper. The $c$ axis orientational function is given by

$$
P_{2}(\theta)=f_{\varepsilon}=1-f_{\alpha}-f_{\beta}
$$

